\title{
Semence, vertu formatrice et intellect agent chez Nicolò Leoniceno entre la tradition arabo-latine et la renaissance des commentateurs grecs
}

\author{
Hiro Hirai* \\ Centre d'histoire des sciences, Université de Gand (Belgique)
}

\begin{abstract}
The treatise On Formative Power (Venice, 1506) of Ferrara's emblematic medical humanist, Nicolò Leoniceno (1428-1524), is the one of the first embryological monographs of the Renaissance. It shows, at the same time, the continuity of medieval Arabo-Latin tradition and the new elements brought by Renaissance medical humanism, namely through the use of the ancient Greek commentators of Aristotle like Simplicius. Thus this treatise stands at the crossroad of these two currents. The present study analyses the range of Leoniceno's philosophical discussion, determines its exact sources and brings to light premises for the early modern development of the concept of formative force, which will end up in the theory of "plastic nature" at the heart of the Scientific Revolution.
\end{abstract}

\section{Keywords}

Nicolò Leoniceno (1428-1524), Renaissance medicine, embryology, psychology, humanism

\section{Introduction}

La théorie de la "nature plastique " du platonicien de Cambridge, Ralph Cudworth (1617-1688), est connue dans l'histoire de la philosophie grâce à l'attention que lui ont prêtée de grands savants de son temps comme G. W. Leibniz. Mais l'histoire de la notion même de

\footnotetext{
*) Je remercie M. Vanhaelen, K. Jadoul, M. Muneo et A. Tawara pour leur aide dans la réalisation du présent article. Ma gratitude cordiale va également à Caroline Leuris ainsi qu’à Nobu et Nancy Siraisi.
} 
"force plastique ", germe de la théorie de Cudworth, demeure jusqu’à présent très peu étudiée. $\mathrm{Si}$, dans sa forme latine, la «vertu formatrice» (virtus formativa) s'observe fréquemment dans les discours embryologiques de la médecine galénique du Moyen Âge, c'est Galien (129-ca. 216) lui-même qui, utilisant souvent les mots "façonner"

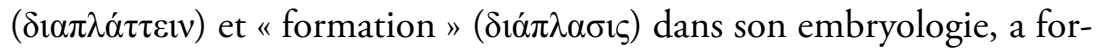

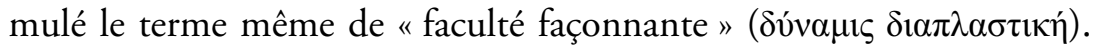
Pour Galien, cette faculté ou force, qui ne peut s'expliquer par les qualités des éléments, est responsable de toute une série d'actions complexes dans la formation du corps des vivants. Pour cette faculté, la plupart des historiens se contentent de continuer à se référer au seul traité Des facultés naturelles, I, 6. Mais il faut remarquer que le corpus entier de Galien compte au moins sept fois l'usage de ce terme, entre autres dans les traités De la semence et Des tempéraments ${ }^{1}$. Bien que la conception même de Galien n'ait pas encore été bien étudiée par les spécialistes, nous pouvons dire que c'est principalement de sa théorie que se sont inspirés les savants occidentaux en ce qui concerne le développement d'idées similaires.

Le médecin persan Avicenne (980-1037), qui a donné une interprétation de cette notion galénique, a surtout favorisé sa réception chez les Latins ${ }^{2}$. Il parle de la "vertu formatrice" (quwwa musawwira) principalement en trois endroits de sa somme médicale extrêmement populaire, Canon. Il s'agit des chapitres sur les facultés naturelles assis-

1) Voir comme texte Karl G. Kühn, Galeni opera omnia, 20 vols (Leipzig, 1821-33; repr. Hildesheim, 1965), désormais indiqué comme K, et l'édition critique de chaque ouvrage, mentionnée dans les notes adéquates. Sur la faculté façonnante, voir Galien, De semine, II, 2 et 5 (K, IV, 611 et 642 = Phillip De Lacy, Galen : On Semen (Berlin, 1998), 162 et 196) ; De temperamentis, II, 6 (K, I, 635-6 = Georg Helmreich, Galeni De temperamentis libri III (Leipzig, 1904), 79-80); De facultatibus naturalibus, I, 6 et II, 3 et 6 (K, II, 15, 86 et 101).

2) Sur l'embryologie d'Avicenne et la tradition arabe, voir l'étude importante mais souvent négligée d'Ursula Weisser, Zeugung, Vererbung und pränatale Entwicklung in der Medizin des arabisch-islamischen Mittelalters (Erlangen, 1983). Cf. Basim Musallam, "Biology and Medicine », dans l'article «Avicenna ", Encyclopedia iranica 3 (1989), 94-9; id., "The Human Embryo in Arabic Scientific and Religious Thought ", in Gordon R. Dunstan (éd.), The Human Embryo : Aristotle and the Arabic and European Traditions (Exeter, 1990), 32-46. 
tées, sur les causes du sperme et sur la génération de l'embryon ${ }^{3}$. À cela, il faut ajouter le traité Des animaux, huitième livre de la partie naturelle de son chef-d'œuvre, Shifâ. Le Des animaux consiste en grande partie en une paraphrase de l'œuvre biologique d'Aristote et a été traduit en latin par Michel Scot (ca. 1175-ca. 1234).

Outre Galien et Avicenne, il faut citer l'intervention du Commentateur, Averroès (1126-1198). Ce dernier mentionne la notion de vertu formatrice des "médecins" non seulement dans son ouvre médicale Colliget (traduit en latin en 1285), II, 10, mais aussi dans ses ouvrages philosophiques tels que le commentaire sur le livre Des animaux et le grand commentaire sur la Métaphysique d'Aristote.

Sous l'autorité de ces auteurs, les Latins ont naturellement accepté la notion de force façonnante sous la forme de "vertu formatrice " (virtus formativa) ou de "vertu informatrice " (virtus informativa), bien que la distinction entre ces deux termes ne soit pas toujours claire à cause du choix obscur de termes opéré dans la traduction latine du Canon $^{4}$. Parmi les savants scolastiques de premier rang, Albert le Grand (ca. 1193-1280) est connu pour son ample usage de la notion de vertu formatrice. Il généralise cette notion et l'applique non seulement au domaine biologique mais aussi à la physique en général y compris la formation des minéraux et des fossiles. Ainsi, dans la tradition scolastique de la discussion sur la génération des choses naturelles, le recours à la vertu formatrice était très fréquent.

Tel est le contexte historique dans lequel s'inscrit l'opuscule De virtute formativa de Nicolò Leoniceno (1428-1524), figure emblématique de la médecine humaniste de Ferrare, que nous allons aborder dans la présente étude ${ }^{5}$. Cet opuscule d'une vingtaine de pages a d'abord été

3) Voir respectivement Avicenne, Canon, I, fen 1, doct. 6, cap. 2 ; III, fen 20, tr. 1, cap. 3 ; III, fen 21, tr. 1, cap. 2 (éd. Giunta, Venise, 1555, ff. 20r ; 372r ; 380v).

4) Voir Giuseppe Michele Nardi, Problemi d'embriologia umana antica e medioevale (Florence, 1938) ; Bruno Nardi, Studi di filosofia medievale (Rome, 1960) ; Romana Martorelli Vico, Medicina e filosofia : per una storia dell'embriologia medievale nel XIII e XIV secolo (Milan, 2002) ; Maaike Van Der Lugt, Le ver, le démon et la vierge : les théories médiévales de la génération extraordinaire (Paris, 2004).

5) Sur sa vie et son œuvre, voir Dictionary of Scientific Biography 8 (1973), 248-50 ; William F. Edwards, "Nicolò Leoniceno and the Origins of Humanist Discussion of Method ", in Edward P. Mahoney (éd.), Philosophy and Humanism : Renaissance Essays 
publié à Venise en 1506, puis réédité en 1524 avec quelques traductions des traités de Galien et enfin recueilli dans l'œuvre réunie de Leoniceno (Bâle, 1532), assurant ainsi une large diffusion transalpine. Ce traité est l'une des premières monographies embryologiques de la Renaissance ${ }^{6}$. Dans cet ouvrage, nous pouvons observer à la fois la continuation de la tradition médiévale arabo-latine et les nouveaux éléments qu'y a apporté l'humanisme renaissant d'orientation philologique. Nous pouvons donc considérer que ce traité se situe au carrefour des deux courants.

Dans une étude précédente, nous avons montré que Jean Fernel (1497-1558), l'un des médecins les plus influents de la Renaissance, s'inspirait largement de cet ouvrage de Leoniceno pour construire sa propre œuvre médico-philosophique De abditis rerum causis (Paris, $1548)^{7}$. C'est sans doute à travers ce dernier traité de Fernel, très lu jusqu'au milieu du dix-septième siècle, que les thèmes abordés par Leoniceno ont exercé un impact important, jusqu’alors insoupçonné, sur les spéculations non seulement embryologiques mais aussi philosophiques de l'époque. Ainsi, l'objet de la présente étude est d'analyser l'étendue de la discussion de Leoniceno, d'en préciser les sources et de mettre à jour les prémisses du développement renaissant de la notion de vertu formatrice, notion qui aboutira, au cœur de la Révolution scientifique, à la théorie de la nature plastique ${ }^{8}$.

in Honor of Paul Oskar Kristeller (Leyde, 1976), 283-305; Daniela Mugnai Carrara, "Profilo di Nicolò Leoniceno ", Interpres 2 (1979), 169-212 ; ead., "Fra causalità astrologica e causalità naturale : gli interventi di Nicolò Leoniceno e della sua scuola sul morbo gallico », Physis 21 (1979), 37-54 ; ead., " Una polémica umanistico-scolastica circa l'interpretazione delle tre dottrine ordinate di Galeno ", Annali dell'stituto e museo di storia della scienza di Firenze 8 (1983), 31-57 ; Vivian Nutton, "The Rise of Medical Humanism : Ferrara, 1464-1555 ", Renaissance Studies 11 (1997), 2-19.

6) Sur ce traité, voir Vivian Nutton, «The Anatomy of the Soul in Early Renaissance Medicine ", in Human Embryo, 136-57, ici 138-40 et 152-3. Nous avons utilisé comme texte l'édition de Venise (1506) et celle des Opuscula (Bâle, 1532), ff. 83v93r. La pagination est indiquée comme suit : éd. (1506) = éd. (1532).

7) Hiro Hirai, "Alter Galenus : Jean Fernel et son interprétation platonico-chrétienne de Galien ", Early Science and Medicine 10 (2005), 1-35, ici 25-26 et 32-33. Cf. Hiro Hirai, Le concept de semence dans les théories de la matière à la Renaissance : de Marsile Ficin à Pierre Gassendi (Turnhout: Brepols, 2005), 83-103.

8) Chez Kircher, voir Hiro Hirai, «Interprétation chymique de la création et origine corpusculaire de la vie chez Athanasius Kircher ", Annals of Science, à paraître. 


\section{Galien : âme végétative et chaleur native}

Dans le De la vertu formatrice, Leoniceno explique d'abord le motif de sa publication. Il a reçu la lettre d'un ami lui demandant, suite à un débat avec un autre savant, les avis de Galien et d'Aristote sur l'identité de la vertu formatrice. À cette occasion, un imprimeur sollicite Leoniceno de publier ses traductions de Galien. Mais en attendant leur parution, pour ne pas faire attendre son ami, l'humaniste de Ferrare décide de publier un opuscule sur la vertu formatrice sous forme d'une lettre ouverte. Il nous avertit que, si cette question enferme "tant d'ambiguïtés presque inexplicables ", c'est parce qu'Aristote et Galien semblent avoir toujours hésité à donner une réponse définitive. Il avoue aussi qu'il aurait pu apporter beaucoup d'éléments écrits par d'autres sur ce sujet mais qu'il a plutôt choisi d'étudier à fond les opinions d'Averroès et de Pietro d'Abano (1257-ca. 1315) le Conciliateur car il estime que leurs opinions exercent une autorité particulièrement importante auprès des philosophes et des médecins de son époque?

Leoniceno débute sa discussion par l'examen de l'avis de Galien exprimé dans le traité De la formation des foetus. Il faut noter que ce dernier ouvrage embryologique était quasi inconnu des Latins. C'est Leoniceno lui-même qui est le premier à l'utiliser à la Renaissance. Il cite longuement la parole de Galien qui avoue ignorer la cause façonnante des foetus. Il s'agit de la conclusion du De la formation des foetus, 6, qui est particulièrement intéressante pour comprendre la pensée elle-même de Galien ${ }^{10}$. Résumons l'essentiel du propos. En admettant une sagesse et faculté suprême dans la formation des foetus, Galien se

9) Sur Pietro d'Abano, voir Sante Ferrari, I tempi, la vita, le dottrine di Pietro d'Abano (Gênes, 1900) ; Bruno Nardi, Saggi sull'aristotelismo padovano dal secolo XIV al XVI (Florence, 1958), 1-74 ; Nancy G. Siraisi, Arts and Sciences at Padua : The Studium of Padua before 1350 (Toronto, 1973) ; Eugenia Paschetto, Pietro d'Abano, medico e filosofo (Florence, 1984); Luigi Olivieri, Pietro d'Abano e il pensiero neolatino : filosofia, scienza e ricerca dell'Aristotele greco tra i secoli XIII e XIV (Padoue, 1988).

10) Galien, De formatione foctuum, 6 (K, IV, 700-2 = Diethard Nickel, Galen: Über die Ausformung der Keimlinge (Berlin, 2001), 105-7). Voir Paul Moraux, "Galien comme philosophe : la philosophie de la nature ", in Vivian Nutton (éd.), Galen : Problems and Prospects (London, 1981), 87-116, ici 114-6; Hirai, "Alter Galenus ", 6-7. Sur l'âme concupiscible, voir Platon, République, IV, 439 D-E, VIII, 550 B; Timée, $70 \mathrm{D}, 77 \mathrm{~B}$. 
demande si l'âme résidant dans la semence en est responsable. Selon lui, les Aristotéliciens appellent cette âme "âme végétative » et les Platoniciens " âme concupiscible » tandis que les Stoöciens l'appellent non pas " âme " mais " nature ". Galien lui-même estime que cette âme est dépourvue de sagesse et totalement irrationnelle. Il admet aussi que, quand l'un de ses maîtres platoniciens lui a enseigné que c'est l'âme du monde qui forme l'embryon, il trouvait possible d'attribuer une telle faculté à cette âme universelle. Mais, pour lui, s'il faut penser que les scorpions et d'autres bêtes détestables sont aussi formés par cette âme, c'est presque un blasphème. Tout ce qu'il peut dire de certain, c'est qu'il existe une telle sagesse suprême comme cause de la formation foetale.

Cet argument fait penser que Galien n'avait pas de certitude sur l'identité de la vertu formatrice. Mais Leoniceno suggère que celui-ci a préféré l'âme végétative qui réside dans la semence. Il sait en effet que Galien présente cette opinion comme celle d'Hippocrate dans le traité De la semence, I, 9-10 $1{ }^{11}$. Là, en comparant la formation du foetus avec celle de la plante, le médecin de Pergame reproche à Aristote d'avoir différemment expliqué l'œuvre de la nature entre les plantes et les animaux. Selon lui, le foetus doit posséder avant tout le principe végétatif qui façonne les parties du corps à partir de la semence. Alors Leoniceno estime que Galien, qui suit fidèlement Hippocrate, conçoit la vertu formatrice comme une puissance de l'âme végétative qui réside dans la semence.

Il faut rappeler qu'à la différence de cette théorie, Galien identifie dans le commentaire des Aphorismes, I, 15, ce qui forme, nourrit et fait croître un animal à la chaleur naturelle ${ }^{12}$. Selon Leoniceno, malgré ces deux conceptions différentes, Galien est cohérent. Il dit :

Que personne ne pense pour cela que [Galien] se contredise lui-même ou par rapport à Hippocrate, à savoir qu'il a attribué la formation de l'animal, d'une part, à l'âme végétative qui est dans la semence comme dans le livre De la semence

11) Galien, De semine, I, 9-10 (K, IV, 542-7 = De Lacy, 93-9). Sur ce traité, voir Hirai, "Alter Galenus », n. 12.

12) Galien, Commentaires sur les Aphorismes d'Hippocrate, I, 15 (K, XVII-B, 420). Sur l'âme comme chaleur, voir Hippocrate, Du régime, I, 10 (Littré, VI, 486) ; Hirai, "Alter Galenus », n. 13. 
et, d'autre part, à la chaleur naturelle comme dans la première partie des Aphorismes. De fait, l'idée que l'âme est la même chose que la chaleur naturelle ou le tempérament du corps, qu'on appelle " complexion », est aussi la doctrine d'Hippocrate $^{13}$.

Pour Leoniceno, bien que Galien avoue ignorer la substance de l'âme et de la cause façonnante des animaux dans le De la formation des foetus, il présente ailleurs, tantôt comme l'opinion d'Hippocrate tantôt comme la sienne, l'idée que l'âme qui forme le fotus est un certain " tempérament (temperamentum), c'est-à-dire un mélange des quatre qualités des éléments ${ }^{14}$. Comme preuve, il puise la parole de Galien dans le traité $D u$ tremblement, 6. Dans ce passage particulièrement important pour son système physiologique, Galien identifie la chaleur naturelle à la "nature " et à l'" âme » dans l'animal et affirme que cette chaleur n'est ni extrinsèque ni postérieure à la naissance de l'animal, mais bien congénitale ${ }^{15}$. Il s'agit là du germe de la célèbre théorie de la " chaleur native" (calor nativus) ou " chaleur innée » $\left(\right.$ calor innatus) ${ }^{16}$. Leoniceno conclut que, si cette chaleur, introduite dans la semence dès le début, est l'âme qui engendre l'animal, Galien est en accord avec Hippocrate. Ainsi, pour lui, la vertu formatrice de Galien doit être une puissance de l'âme végétative, identifiée à la chaleur innée ou à un certain tempérament.

13) De virtute, f. $2 \mathrm{v}=$ f. $84 \mathrm{v}$ : [Galenus] quem nemo putet ob id a seipso, aut ab Hippocrate dissentire, quod aliquando animalis formationem animae tribuit vegetali, quae in semine existit, veluti in libro De semine: aliquando calori naturali, ut in prima particula Aphorismorum. Nam hoc quoque Hippocratis est dogma, eandem rem esse animam et calorem naturalem, sive corporis temperaturam quam complexionem vocant.

14) Sur la notion du temperamentum, voir Hirai, "Alter Galenus ", n. 14.

15) Galien, De tremore, 6 (K, VII, 616 = David Sider et Michael McVaugh, "Galen On Tremor, Palpitation, Spasm, and Rigor: Galeni De tremore, palpitatione, convulsione, et rigore ", Transactions and Studies of the College of Physicians of Philadelphia, 5th series 1 (1979), 183-210, ici 199-200).

16) Sur la chaleur native, voir Everett Mendelsohn, Heat and Life : The Development of the Theory of Animal Heat (Cambridge MA, 1964); Thomas S. Hall, "Life, Death and the Radical Moisture: A Study of Thematic Pattern in Medieval Medical Theory », Clio Medica 6 (1971), 3-23 ; Peter H. Niebyl, "Old Age, Fever, and the Lamp Metaphor ", Journal of the History of Medicine 26 (1971), 351-68 ; Michael McVaugh, "The humidum radicale in Thirteenth-Century Medicine ", Traditio 30 (1974), 25983. 


\section{Aristote et Pietro d'Abano : chaleur céleste, intellect et véhicule de l'âme}

Leoniceno aborde ensuite l'opinion d'Aristote. Il nous avertit que celui-ci traite souvent des questions difficiles à la manière de la seiche qui noircit de l'eau pour ne pas être capturée. Il propose dès lors de se fonder également sur les écrits de ses commentateurs. Tout d'abord, il rappelle le témoignage de Galien qui affirme dans le traité Des tempéraments, II, 6, qu'Aristote a douté de l'identité de la vertu formatrice. En effet, Galien critique dans ce passage ceux qui n'ont pas considéré la cause de la formation animale comme la vertu formatrice, qui rend habilement les parties du corps conformes aux mœurs de l'âme. Il ajoute qu'Aristote s'est demandé s'il ne fallait pas attribuer cette vertu à un principe plus divin que les quatre qualités élémentaires ${ }^{17}$. Mais Galien ne précise pas, nous le savons, l'endroit où Aristote a exprimé ce doute. Quant à Leoniceno, il pense qu'il s'agit de la fin du traité des Météorologiques, IV, 12. Là, Aristote attribue la cause de la formation des parties homéomères au chaud et au froid en évoquant la "nature" ou "une autre cause " pour la formation des parties anhoméomères, c'est-à-dire des organes, comme la tête et le pied, qui sont construits à partir des homéomères ${ }^{18}$. Selon Leoniceno, c'est suivant cette distinction que Galien établit deux sortes de vertus génératrices dans le Des facultés naturelles, I, $6: 1$ ) la "vertu altératrice " (virtus alterativa) qui est la première vertu mutatrice et agit à travers la chaleur ; 2) la « vertu formatrice" (virtus formativa) qui est l'art suprême et agit par l'ordre de son Créateur. Leoniceno suit toutefois ici l'interprétation qu'Avicenne expose dans son Canon sur la base de la théorie de Galien. Ainsi, la vertu altératrice agit par les qualités élémentaires et façonne l'os, le nerf et d'autres parties homéomères tandis que la vertu formatrice établit l'assemblage des membres organiques (selon Galien) et façonne le trait et la figure des membres en effectuant d'autres opérations com-

17) Galien, De temperamentis, II, 6 (K, I, 635-6 = Georg Helmreich, Galeni De temperamentis libri III (Leipzig, 1904), 79). Cf. De temperamentis, I, 9 (K, I, $567=\mathrm{Hel}-$ mreich, 36).

18) Aristote, Météorologiques, IV, 12, 390 b 3-15. 
plexes (selon Avicenne) ${ }^{19}$. Pour Leoniceno, c'est à propos de la deuxième vertu qu'Aristote et Galien se demandaient si elle n'était pas plus divine que la force des qualités des éléments.

Après cette explication, Leoniceno déplore que plusieurs philosophes et médecins aient recours, suivant la tradition des Arabes, à une autre phrase d'Aristote pour chercher la nature de la vertu formatrice. Il s'agit du passage énigmatique du traité De la génération des animaux (désormais indiqué comme $G A$ ), II, 3, qui a une dimension particulièrement cosmologique. Le voici d'après la traduction moderne :

Il y a toujours dans le sperme ce qui rend les semences fécondes, c'est-à-dire ce qu'on appelle " chaleur ». Or cette chaleur n'est ni du feu ni une substance de ce genre, mais le pneuma emmagasiné dans le sperme et dans l'écumeux, et la nature inhérente à ce pneuma et qui est analogue à l'élément astral. C'est pourquoi le feu n'engendre aucun animal, et il est manifeste qu'aucun être ne se forme dans les matières du feu, qu'elles soient humides ou sèches. Au contraire la chaleur solaire a le pouvoir d'engendrer ainsi que la chaleur animale, non seulement celle qui se manifeste par le sperme, mais s'il se produit quelque autre résidu naturel, il ne possède pas moins lui aussi un principe vital. Il ressort donc de ce qui précède que la chaleur qui réside dans les animaux n'est pas du feu et qu'elle n'a pas son principe dans le feu ${ }^{20}$.

C'est à propos de ce passage que les Arabes comme Avicenne et Averroès ont parlé de la vertu formatrice et que leur successeur Pietro d'Abano, parmi les Latins, a développé une interprétation bien particulière $^{21}$. Leoniceno remarque qu'Aristote, en parlant de la chaleur de la semence, n'exprime pas d'hésitation telle que Galien l'a décrite. Pour

19) Galien, De facultatibus naturalibus, I, 6 (K, I, 12 = Charles Daremberg, Euvres anatomiques, physiologiques et médicales de Galien (Paris, 1854), I, 219-20) ; Avicenne, Canon, I, fen 1, doc. 6, cap. 2 (éd. Giunta, Venise, 1555, f. 20r).

20) Aristote, De la génération des animaux, II, 3, 736 b 33-737 a 7 (trad. P. Louis). Cf. Paul Moraux, "À propos du võ̃ $\theta$ v́ $\rho \alpha \theta \varepsilon v$ ", in Augustin Mansion (éd.), Autour d'Aristote (Louvain, 1955), 255-95 ; David M. Balme, Aristotle's De partibus animalium $I$ and De generatione animalium I (Oxford, 1972), 161-4 ; Gad Freudenthal, Aristotle's Theory of Material Substance: Heat and Pneuma, Form and Soul (Oxford, 1995), 116-9.

21) Avicenne, De animalibus, XVI, i (éd. Venise, 1508 ; repr. Louvain, 1961, f. 61va); Averroès, Commentaire sur le Des animaux, XVI, 3 (éd. Giunta, Venise, 1562, VI, f. 75vb) ; Pietro d'Abano, Conciliator, diff. 48 (éd. Giunta, Venise, 1565 ; repr. Padoue, 1985, f. 72). Cf. Gad Freudenthal, "The Medieval Astrologization of Aristotle's Biol- 
lui, le Stagirite ne fait pas ici d'allusion à la vertu formatrice. En comparant la génération spontanée causée par la chaleur céleste avec la génération effectuée par la chaleur séminale, Leoniceno explique alors la véritable nature de cette chaleur séminale :

En effet, Aristote ne nie pas que ces qualités, c'est-à-dire l'humide et le sec, contribuent en tant que principes passifs à la constitution des animaux, lorsque la chaleur vivifiante, qu'il affirme analogue à la chaleur des étoiles, agit en ces [qualités]. Telle est la chaleur qui est contenue dans la semence. Car comme la chaleur céleste agit sur la boue d'où elle engendre des animaux, la chaleur de la semence effectue la même chose dans la matière qui lui convient. C'est pourquoi Aristote n'appelle pas cette chaleur introduite dans la semence " chaleur céleste ", comme certains le pensent, mais " analogue à la chaleur céleste »"

En insistant sur le fait que la chaleur séminale est seulement " analogue " à la chaleur céleste pour Aristote, Leoniceno nie l'identification que Pietro établit entre ces deux chaleurs. Il divise alors la chaleur en deux sortes : l'une est utilisée par la nature comme instrument pour la génération animale et l'autre l'est par l'art pour la perfection des œuvres. Pour Leoniceno, la première chaleur, instrument de la génération animale, est contenue dans la semence et possède un principe vital selon la phrase d'Aristote. Il précise que cette chaleur vivifiante n'est pas seulement enfermée dans la semence mais aussi dans des résidus sordides. Pour lui, aucune personne saine d'esprit ne peut estimer " céleste" ou "divine " la chaleur contenue dans de tels résidus. Il accuse dès lors Averroès et Pietro d'avoir conclu, en se fondant sur ce passage, qu'Aristote assimile la vertu formatrice aux principes divins en l'appelant "chose divine " ou "intellect " et en l'estimant "séparable du corps ".

ogy: Averroes on the Role of the Celestial Bodies in the Generation of Animate Beings ", Arabic Sciences and Philosophy 12 (2002), 111-37.

22) De virtute, f. $3 \mathrm{r}=$ f. $86 \mathrm{r}$ : Has enim qualitates, scilicet humiditatem et siccitatem tamquam principia passiva conferre ad constitutionem animalium, non negat Aristoteles : quum calor vivificus agit in ipsas, quem ait esse proportionalem calori stellarum, qualis est qui in semine continetur. Sicuti enim calor cœlestis agens in lutum, ex eo generat animalia, ita et calor seminis in materiam sibi convenientem idem operatur. Quare hic calor semini inditus non calor cœlestis, ut quidam opinantur, sed calori cœlesti poportionalis ab Aristotele dicitur. 
À partir d'ici, Leoniceno se concentre sur la critique de la différence, 48, du Conciliator, chapitre important pour l'embryologie de Pietro. Pour lui, celui-ci déforme complètement ce qu'Aristote a enseigné. Résumons l'essentiel du passage que Leoniceno tire de Pietro ${ }^{23}$. Comme la vertu formatrice ne semble pas avoir d'instrument figuré dans la masse spermatique, Aristote estime cette vertu "séparable » du corps, bien que sa séparabilité soit beaucoup plus faible que celle de l'intellect totalement séparé de la matière. Pour Pietro, la vertu formatrice est divine, agissante et supérieure aux autres vertus génératrices puisqu'elle est simple et presque immatérielle. Mais comme elle est incapable de toucher directement la matière, elle a besoin d'un substrat distinct qui peut la véhiculer pour la génération du foetus. Ce véhicule est un corps subtil, spiritus. La vertu formatrice a aussi besoin des deux instruments dont l'un est la chaleur céleste qui est toujours vivifiante et jamais destructive en faisant accéder les êtres produits à une certaine conformité avec le ciel. Elle est l'instrument le plus digne de tous et, par son intermédiaire, la vertu formatrice exerce ses actions nobles qui déterminent tel ou tel être. L'autre instrument de la vertu formatrice est la chaleur élémentaire qui ne peut vivifier et conserver des choses naturelles qu'avec l'aide de la chaleur céleste. Enfin, Pietro ajoute que Galien a également recours à cette explication de la double chaleur en ce qui concerne la maturation des céréales dans son traité Des facultés des médicaments simples, IV, $12^{24}$.

Bien qu'il estime la réputation de Pietro considérable, Leoniceno ose le contredire. Il critique tout d'abord l'idée de Pietro sur le double instrument de la vertu formatrice (chaleur céleste et chaleur ignée). Pour Leoniceno, cette idée n'est pas cohérente avec l'enseignement d'Aristote. En effet, même selon la traduction médiévale que Pietro utilise, Aristote montre dans ce passage que la chaleur céleste n'est pas

23) Pietro d'Abano, Conciliator, diff. 48 (Giunta, f. 72, col. 1 A-C). Cf. Nardi, Saggi sull'aristotelismo, 3-8. Voir aussi Paschetto, Pietro d'Abano, 199-202 ; Danielle Jacquart, "Médecine et astrologie à Paris dans la première moitié du XIVe siècle ", in Graziella Federici Vescovini et al. (éds.), Filosofia, scienza e astrologia nel Trecento europeo (Padoue, 1992), 121-34 ; ead., "L'influence des astres sur le corps humain chez Pietro d'Abano ", in Bernard Ribémont (éd.), Le corps et ses énigmes au Moyen Age (Caen, 1993), 73-86.

24) Galien, De simplicium medicamentorum facultatibus, IV, 12 (K, XI, 657). 
l'instrument de la vertu formatrice ou de toute autre vertu qui la dirige. Leoniceno souligne en particulier que le Stagirite place la chaleur, qui féconde la semence, à l'intérieur de la semence et non à l'extérieur comme la chaleur céleste. Il précise aussi que la phrase " cette chaleur n'est ni du feu ni une vertu de ce genre " est ajoutée pour que personne ne l'identifie faussement à la chaleur ignée qui n'est pas génératrice mais destructrice. La chaleur séminale n'est donc ni céleste ni ignée. C'est pourquoi Aristote estime que la nature génératrice de cette chaleur, qui est contenue dans la semence ou dans le spiritus de celle-ci, est seulement "analogue " au rang des astres. Car ce qui est analogue n'est pas identique. Leoniceno cite, à l'appui de cette interprétation, la nouvelle traduction de Théodore de Gaza (1400-1476) qui se lit comme suit : "correspondant par analogie à l'élément des étoiles $"^{25}$.

Leoniceno explique alors comment comprendre correctement la chaleur séminale. Selon lui, il existe une troisième chaleur qui, bien qu'elle soit issue de l'élément de feu, est propre à la génération des vivants et est estimée, pour cette raison, analogue à la chaleur céleste. Il nous avertit de ne pas considérer la chaleur céleste comme la cause de la génération animale suivant à tort la célèbre phrase d'Aristote, Physique, II, 2: "Ce qui engendre l'homme, c'est l'homme plus le soleil ${ }^{26}$. Il refuse surtout de considérer la chaleur céleste comme l'instrument interne de la semence et de la lier à la vertu formatrice. Puis il explique ce que Galien voulait dire dans le passage du Des facultés des médicaments simples sur lequel Pietro fondait son argument. Selon lui, outre la chaleur interne qui est conçue comme l'âme des semences, Galien admettait la chaleur céleste comme auxiliaire nécessaire pour la maturation des céréales.

Ensuite Leoniceno aborde la question de savoir si Aristote appelle la vertu formatrice « intellect» ou " chose divine » et s'il l'estime « sépa-

25) Sur Théodore de Gaza, voir John Monfasani, George of Trebizond: A Biography and a Study of his Rhetoric and Logic (Leyde, 1976) ; id., "The Pseudo-Aristotelian Problemata and Aristotle's De Animalibus in the Renaissance ", in Anthony Grafton et Nancy Siraisi (éds.), Natural Particulars: Nature and the Disciplines in Renaissance Europe (Cambridge MA, 1999), 205-47.

26) Aristote, Physique, II, 2, 194 b 14. Cf. Aristote, De la génération et de la corruption, II, 10,336 a 31 . 
rable du corps". Selon lui, quand le Stagirite énonce que "l'intellect seul vient du dehors ", que "c'est seulement lui qui est divin » et que " cela n'a rien de commun avec le corps", il ne parle que de l'intellect (mens ou intellectus $)^{27}$. Si ces trois phrases concernent le seul intellect, comment pourraient-elles aussi s'appliquer à la vertu formatrice ? En estimant la nouvelle traduction de Théodore plus fidèle et plus claire, Leoniceno affirme que ces phrases ne parlent pas de la vertu formatrice mais seulement de ce qui est appelé « intellect ». Et il donne une autre phrase cruciale d'Aristote qui parle aussi de l'intellect ( $G A, 737$ a $7-$ 12) :

Or le corps du sperme [est ce] dans lequel est véhiculée la semence du principe psychique, en partie séparable du corps chez les êtres où est comprise une partie divine telle que celle qui est appelée « intellect », en partie inséparable, etc. ${ }^{28}$

Si chacune de ces quatre phrases pouvait s'appliquer à la vertu formatrice comme le veut Pietro, les caractères tels que l'identité avec l'intellect, la séparabilité, la divinité, l'origine extrinsèque et la résidence dans la semence seraient aussi attribués à cette vertu. Mais Leoniceno affirme que tout cela est faux. Pour lui, Pietro lui-même aurait dû s'en apercevoir. Pourtant celui-ci, à cause de l'obscurité de la traduction "barbare", a inventé un terme étonnant auquel Aristote n’a jamais songé. Car il a non seulement identifié la vertu formatrice à l'intellect mais aussi l'a nommée "intellect appelé " (intellectus vocatus) à la différence de l'intellect passif ou actif. Mais dans quel texte

27) Aristote, De la génération des animaux, II, 3, 736 b 27-9. Leoniceno utilise les deux termes, mens et intellectus, comme synonymes. Sur l'idée de l'intellect dans la tradition arabo-latine, voir Étienne Gilson, «Les sources gréco-arabes de l'augustinisme avicennisant ", Archives d'histoire doctrinale et littéraire du Moyen Âge 4 (192930), 5-149 ; Herbert A. Davidson, Alfarabi, Avicenna, and Averroes, on Intellect: Their Cosmologies, Theories of the Active Intellect, and Theories of Human Intellect (Oxford, 1992).

28) De virtute, $\mathrm{f} .3 \mathrm{v}=\mathrm{f} .87 \mathrm{r}$ : Corpus autem geniturae in quo semen animalis principii contentum una provenit, partim separabile a corpore in quibus divina pars comprehenditur, qualis est quae mens appellatur : partim inseparabile, etc. Cf. Aristote, De la génération des animaux, II, 3, 737 a 7-12. Voici la version médiévale que Pietro donne dans le Conciliator, diff. 48 (Giunta, f. 72, col. 1 A) : Geniturae corpus, in quod egreditur, quod animalis principii. Et hoc quidem separabile existens a corpore in quibuscumque apprehenditur quid divinum. Talis autem [est] quod vocatus intellectus. 
d'Aristote, se demande Leoniceno, Pietro a-t-il trouvé le terme "appelé " (vocatus) pour désigner une propriété de l'intellect comme " passif » et " actif » ? Pour l'humaniste ferrarais, ce n'est qu'une sottise. Il conclut que la nouvelle traduction de Théodore, qui adopte la phrase "une partie divine telle que celle qui est appelée "intellect" etc.", détruit l'identification erronée de Pietro.

Enfin Leoniceno s'attaque à la première partie de l'argument de Pietro qui affirmait que le Stagirite estime "séparable " la vertu formatrice puisqu'elle n'a pas d'instrument figuré dans le sperme. Selon Leoniceno, si une vertu est estimée "séparable du corps", ce n'est pas parce qu'elle n'a pas d'instrument figuré, mais bien parce qu'elle n'a pas d'instrument corporel ou, pour utiliser les termes d'Aristote, parce que son action n'a rien de commun avec celle du corps. Il ne s'agit que de la vertu intellectuelle. Les autres puissances de l'âme ne peuvent pas s'exercer sans le corps puisqu'elles en sont inséparables et que leur action est corporelle. À ce propos, Leoniceno a recours à la paraphrase de Thémistius (ca.317-ca. 388) du traité De l'âme, I, 1, où Aristote se demande si l'âme est séparable du corps. D'après Thémistius, s'il n'y a rien de propre à la seule âme et si le corps est toujours requis pour les actions de l'âme, et bien que ce corps soit corporel ou quelque chose de plus intime et plus caché tel que Platon l'imaginait, l'âme ne peut pas subsister séparément du corps ${ }^{29}$. Alors tout en admettant que la vertu formatrice possède un corps subtil, spiritus, comme son instrument, Leoniceno nie que cette vertu soit séparable de son instrument corporel.

Leoniceno refuse aussi la divinité à la vertu formatrice en remarquant que celle-ci est contenue dans un corps sordide, c'est-à-dire dans la semence qui n'est que le résidu de l'aliment. Pour lui, il est absurde

29) Thémistius, Paraphrase du De l'âme, I, 1 (Richard Heinze, Themistii in libros Aristotelis de anima paraphrasis (Berlin, 1899), $6=$ Ermolao Barbaro, Themistii libri paraphraseos (Venise, 1499 ; repr. Francfort, 1978), f. 69r = Robert Todd, Themistius : On Aristotle On the Soul (London, 1996), 20). La partie "tel que Platon l'imaginait" semble l'interpolation d'Ermolao Barbaro (1454-1493). Sa traduction latine a été publiée à Trévise en 1481 et puis plusieurs fois à Venise. Cf. Vittore Branca, «Ermolao Barbaro and Late Quattrocento Venetian Humanism ", in John R. Hale (éd.), Renaissance Venice (London, 1973), 213-43. Sur Thémistius à la Renaissance, voir Robert Todd, "Themistius", Catalogus translationum et commentariorum 8 (2003), 59-102. 
d'attribuer quelque chose de divin aux choses sordides. C'est pourquoi Aristote ne pense pas que la vertu intellectuelle, chose divine, vienne avec la semence mais qu' elle "vient du dehors ". Leoniceno en conclut que cette vertu intellectuelle n'est pas formatrice parce qu'Aristote l'estime tellement séparée du corps qu'elle ne peut pas utiliser comme instrument un corps subtil, même d'un moindre degré de corporéité.

C'est ainsi que Leoniceno achève sa réfutation de Pietro. Mais pour éclaircir davantage le problème des passages évoqués du $G A$, il a recours à un autre texte, l'unique commentaire grec existant de ce traité. Il s'agit du texte parfois attribué faussement à Jean Philopon (ca. 490-ca. 570) mais en réalité composé à Byzance, dans la première moitié du douzième siècle, par Michel d'Éphèse ( $f$. ca. 1138) qui voulait combler la lacune des commentaires sur l'œuvre d'Aristote ${ }^{30}$. Pour montrer que le passage évoqué ( $G A, 737$ a 7-12) concerne uniquement l'intellect, Leoniceno cite le commentaire de Michel qui a rendu, selon lui, la phrase plus harmonieuse, en ajoutant un relatif cujus, ce qui donne le passage suivant :

Mais le corps du sperme [est ce] dans lequel vient ensemble la semence du principe de l'âme. La partie de cette âme, c'est-à-dire l'intellect, est séparée comme ce qui vient du dehors tandis que ce qui est inséparable est, évidemment, la partie irrationnelle ${ }^{31}$.

Pour lui, cette interprétation, qui ne divise que l'âme en partie intellectuelle et en partie irrationnelle, montre clairement qu'Aristote ne parle pas ici de la vertu formatrice.

30) Voir Anthony Preus, Aristotle and Michael of Ephesus on the Movement and Progression of Animals (Hildesheim, 1981) ; id., "Michael of Ephesus and the History of Zoology ", in Aldo Bernardo et Saul Levin (éds.), The Classics in the Middle Ages (Binghamton, 1990), 265-82 ; Robert Browning, "An Unpublished Funeral Oration on Anna Comnena ", in Richard Sorabji (éd.), Aristotle Transformed: The Ancient Commentators and their Influence (London, 1990), 393-406, ici 399-401.

31) De virtute, f. $4 \mathrm{r}=$ f. $87 \mathrm{v}$ : Geniturae autem corpus, in quo una provenit semen principii animae, cujus scilicet animae hoc quidem est separatum, veluti qui deforis intellectus : hoc vero inseparabile, scilicet pars irrationalis. Cf. Michel d'Éphèse, Commentaire sur le De la génération des animaux, II, 3 (Michael Hayduck, Joannis Philoponii (Michaelis Ephesii) in libros de generatione animalium commentaria (Berlin, 1903), 87). 
Leoniceno trouve une autre interprétation chez le cardinal Bessarion $(1403 / 8-1472)^{32}$. Selon lui, celui-ci explique dans son traité In calumniatorem Platonis (Rome, 1469) le sens des passages concernés afin de prouver qu'Aristote est en accord avec Platon sur l'union de l'âme avec le corps. Chez Bessarion, cette union s'effectue par un certain corps intermédiaire de nature excellente, tel que les Platoniciens le décrivent comme le "véhicule " (ŏ $\chi \mu \alpha)$ de l'âme, qui est produit en étant resserré du corps brillant et éthéré3 ${ }^{3}$. Leoniceno ajoute que la notion de ce véhicule a fait l'objet des moqueries d'un accusateur de Platon, c'est-à-dire Georges de Trébizonde (1395-1472/3). Selon Bessarion, qui argumente contre celui-ci, Aristote pensait : 1) qu'un intermédiaire est nécessaire pour cette union des deux extrémités, âme et corps ; 2) que cet intermédiaire est un corps différent, séparé et plus divin que les quatre éléments ; 3) que la nature d'un tel corps differe selon la noblesse et la bassesse de chaque âme. Pour Bessarion, Aristote identifie ce corps au spiritus enfermé dans la semence féconde et estime la nature de ce spiritus analogue à l'élément des étoiles. Ce corps est en partie séparé et en partie non séparé du sperme. Sa partie inséparable est un liquide crasseux et matériel qui se disperse dans le sperme comme la présure dans le lait. Ainsi Bessarion conclut qu'il n'y a aucune raison de se moquer du fait que Platon pense que l'âme vient avec un tel corps dans la génération. Leoniceno évalue, à son tour, l'interprétation du cardinal comme suit :

Or cette explication de la phrase d'Aristote ne diffère pas de la première explication citée de Michel d'Éphèse, si ce n'est en ce que l'un attribue les différences

32) Cf. Ludwig Mohler, Kardinal Bessarion als Theologe, Humanist und Staatsmann, 3 toms. (Paderborn, 1923-42) ; James Hankins, Plato in the Italian Renaissance (Leyde, 1990), 208-63 ; John Monfasani, Byzantine Scholars in Renaissance Italy: Cardinal Bessarion and Other Emigrés (Aldershot, 1995).

33) Bessarion, In calumniatorem Platonis, III, xxii, 3 (Mohler, II, 369). Sur le véhicule de l'âme, voir Eric R. Dodds, Proclus: The Elements of Theology (Oxford, 1933/1963), 315-21 ; Daniel P. Walker, "The Astral Body in Renaissance Medicine », Journal of the Warburg and Courtauld Institutes 21 (1958), 119-33 ; John F. Finamore, Iamblichus and the Theory of the Vehicle of the Soul (Chico, 1985) ; Henry J. Blumenthal, "Soul Vehicle in Simplicius ", in Stephen Gersh (éd.), Platonism in Late Antiquity (Indiana, 1992), 173-88 ; Maria di Pasquale Barbanti, Ochema-Pneuma e phantasia nel neoplatonismo (Catania, 1998). 
séparables et inséparables aux parties de l'âme, c'est-à-dire à la partie intellectuelle et à la partie irrationnelle tandis que l'autre [les attribue] au corps qui est le véhicule de l'âme, qu'Aristote affirme séparable chez les êtres où une partie divine est comprise, comme si l'autre [partie], c'est-à-dire irrationnelle et concupiscible, avait un véhicule et substrat plus crasseux et inséparable du corps corruptible ${ }^{34}$.

À propos de cette théorie néoplatonicienne du véhicule de l'âme, Leoniceno n'oublie pas d'ajouter un passage crucial de Thémistius formulé dans sa paraphrase du De l'âme, I, 3, et destiné à démontrer l'accord d'Aristote avec Platon ${ }^{35}$. Selon Thémistius, ces deux hommes ont enlevé de l'âme tout ce qui est matériel, en lui attribuant un certain corps divin et céleste qui produit l'âme et l'intellect. Il faut bien remarquer que ce commentateur relie manifestement la notion de véhicule de l'âme à la théorie aristotélicienne de la nature éthérée du spiritus enfermé dans la semence. Leoniceno, quant à lui, l'explique en ces termes :

De fait, [Thémistius] montre que ce véhicule raréfié et splendide chez Platon [n'est] rien d'autre qu'un génie duquel résulterait l'animus. Chez Aristote aussi, on trouve une certaine nature attribuée à l'âme, nature qui correspondrait par analogie au cinquième élément et qu'il a estimée appartenant à l'âme de tous les vivants. Donc, selon ces hommes proches de l'école non moins aristotélicienne que platonicienne, il semble que, dans ce passage du deuxième livre De la génération des animaux [...], Aristote ne parle pas de la vertu formatrice mais du véhicule de l'âme qui serait en partie séparable et en partie inséparable ${ }^{36}$.

34) De virtute, f. $4 \mathrm{r}=$ f. $88 \mathrm{r}$ : Non differt autem haec expositio verborum Aristotelis a prima Michaelis Ephesii recitata, nisi in eo quod separabiles atque inseparabiles differentias altera partibus animae tribuit, scilicet intellectuali et irrationali, altera corpori quod est vehiculum animae, quod Aristoteles esse separabile dicit, in quibus pars divina comprehenditur, quasi altera, scilicet irrationalis et appetitiva vehiculum habeat, atque subjectum crassius, et a corpore hoc corruptibili inseparabile.

35) Thémistius, Paraphrase du De l'âme, I, 3 (Heinze, $19=$ Barbaro f. $72 \mathrm{v}=$ Todd, 35). Cf. Platon, Timée, $41 \mathrm{E}$; $44 \mathrm{E}$; 69 C. Ici aussi, la traduction latine de Barbaro semble s'écarter de l'original grec.

36) De virtute, f. $4 \mathrm{r}=$ f. $88 \mathrm{r}$ : Nam apud Platonem vehiculum illud rarum ac splendidum nihil aliud, quam ingenium e quo animus constet, ostendit. Apud Aristotelem quoque naturam quandam animae tributam invenias quae quinto corpori proportione respondeat, quam ad omnium animalium animas pertinere censuit. Videtur ergo Aristoteles secundum istos viros, non minus Aristotelicae quam etiam Platonicae sectae familiares, loco illo libri secundum De generatione animalium [...], non de vir- 
Ce développement est particulièrement intéressant non seulement pour comprendre la pensée de Leoniceno mais aussi celle de Thémistius lui-même parce que certains spécialistes ne veulent pas voir chez celui-ci l'influence du néoplatonisme ${ }^{37}$. Quoi qu'il en soit, nous pouvons dire que le passage de Thémistius offrait une belle occasion de lecture néoplatonisante de sa pensée. Il faut rappeler que Galien relie lui aussi le véhicule de l'âme au cinquième élément d'Aristote dans son traité Des doctrines d'Hippocrate et de Platon, VII, ${ }^{38}$. Mais Leoniceno, qui connaissait certainement ce traité, n'y fait pas allusion. Il se demande tout simplement si un tel véhicule lucide et éthéré, qui appartient à l'âme rationnelle, peut être contenu dans la semence. Mais il ne va pas plus loin et propose de laisser cette question à ceux qui étudient tant le platonisme que l'aristotélisme. En estimant qu'il suffit d'avoir noté des éléments qui contribuent à éclaircir ces passages difficiles du $G A$, il conclut que ceux-ci ne concernent pas la vertu formatrice contrairement à ce que pensait Pietro.

\section{Alexandre d'Aphrodise et Simplicius : nature interne de la semence}

Après avoir ainsi réfuté l'interprétation de Pietro, Leoniceno tente de reconstituer la notion de la vertu formatrice dans la perspective aristo-

tute loqui formativa, sed de vehiculo animae, quod partim sit separabile, et partim inseparabile.

37) Voir Henry J. Blumenthal, "Themistius : The Last Peripatetic Commentator on Aristotle?", in Aristotle Transformed, 113-23 ; id., Aristotle and Neoplatonism in Late Antiquity: Interpretations of the De anima (Ithaca, 1996). Pour le néoplatonisme de Thémistius, voir Edward P. Mahoney, "Themistius and the Agent Intellect in James of Viterbo and Other Thirteenth Century Philosophers (Saint Thomas, Siger of Brabant and Henry Bate ", Augustiniana 23 (1973), 422-67 ; id., "Neoplatonism, the Greek Commentators and Renaissance Aristotelianism », in Dominic J. O’Meara (éd.), Neoplatonism and Christian Thought (Albany, 1982), 169-77 et 264-83 ; Omer

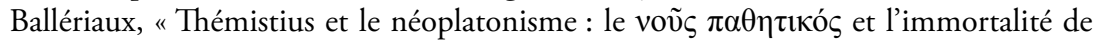
l'âme ", Revue de philosophie ancienne 12 (1994), 171-200 ; id., "Eugénios, père de Thémistios et philosophe néoplatonicien ", L'antiquité classique 65 (1996), 135-60.

38) Galien, De placitis Hippocratis et Platonis, VII, 7 (K, V, 643 = Phillip De Lacy, Galen: On the Doctrines of Hippocrates and Plato (Berlin, 1980), 474). Sur ce traité, voir Hirai, "Alter Galenus ", n. 25. 
télicienne à travers un autre passage du De la formation des foetus, 6, où Galien parle de la "nature" de la semence ${ }^{39}$. Selon celui-ci, les philosophes s'accordent pour penser que la cause de la formation foetale est ce qu'ils appellent "nature " bien que sa substance soit inconnue. Face à la sagesse suprême du Créateur du corps vivant, Galien somme ces philosophes de lui donner la véritable identité de cet artisan par les questions suivantes : est-ce un Dieu sage et puissant qui a préconçu le corps à former pour chaque animal ? ; a-t-il ajouté une faculté afin de le façonner tel qu'il l'avait conçu en esprit ? ; est-ce finalement un Dieu ou une âme autre que divine? Pour Galien, cette nature, qu' elle soit corporelle ou incorporelle, atteint le sommet de la sagesse bien que les philosophes refusent de lui attribuer une telle sagesse. Mais comme ils rejettent aussi l'opinion d'Épicure, selon laquelle tout est fait sans providence, il n'y a que deux options, dit Galien, pour que la formation fotale arrive au meilleur but : 1) par un mouvement privé de raison et d'art ; 2) par un mécanisme comparable à celui des automates. Ce dernier mode est réalisé par un opérateur qui donne le premier mouvement à l'une des pièces. Bien que l'opérateur ne touche plus rien, les automates sont mus pendant un certain temps. Galien estime que seule la deuxième option est digne d'un examen approfondi.

En se fondant sur cet argument de Galien, Leoniceno met la nature contenue dans la semence au cœur de la discussion. Par la nature irrationnelle de la semence, Galien semble songer à la théorie stoïcienne, comme il l'affirme à la fin du De la formation des foetus, $6^{40}$. Pourtant, selon Leoniceno, ce sont bien Aristote et Alexandre d'Aphrodise ( $f$. ca. 200) qui sont ici désignés par le nom de "philosophes». Pour lui, Galien estime que ces hommes renvoient la cause de la génération animale à une certaine puissance irrationnelle appelée " nature " et qu'ils comparent les mouvements de celle-ci à ceux des automates ${ }^{41}$. Or,

39) Galien, De formatione foetuum, 6 (K, IV, 687-9 = Nickel, 90-4).

40) Galien, De formatione fotuum, 6 (K, IV, 700 = Nickel, 104). Cf. Plutarque, Moralia, $1052 \mathrm{~F}=$ SVF, II. 806 ; Galien, De foetuum formatione, 3 (K, IV, 665 = Nickel, 68 = SVF, II. 712).

41) Sur la relation de Galien avec Alexandre, voir Pier Luigi Donini, "Lanima e gli elementi nel De anima di Alessandro di Afrodisia ", Atti della accademia di Torino (Classe di scienze morali, storiche e flologiche) 105 (1971), 61-107, ici 98-107 ; Vivian Nutton, "Galen in the Eyes of his Contemporaries ", Bulletin of the History of Medi- 
selon Aristote, le père introduit dans la semence une certaine force motrice qui meut la matière, sang menstruel que la mère fournit. Le premier mouvement met en action le deuxième, le deuxième le troisième et ainsi de suite jusqu'à la fin de la génération d'un animal complet. C'est là qu'Aristote introduit le modèle des automates ${ }^{42}$. Pour Leoniceno, c'est à propos de ce développement que Galien établit son argument et qu'Alexandre considère la "nature " de la semence comme une puissance irrationnelle. Malheureusement les commentaires d'Alexandre sur l'œuvre d'Aristote n'existent plus ni sur le $G A$ ni sur la Physique. Leoniceno se tourne alors vers le commentaire de Simplicius (fl. 529- ?) sur la Physique d'Aristote, qui contient des fragments du commentaire perdu d'Alexandre sur la théorie de la génération naturelle $^{43}$. Il expose les avis d'Alexandre et de Simplicius pour clarifier l'identité de la vertu formatrice.

Leoniceno cite d'abord un extrait d'Alexandre ${ }^{44}$. Ce fragment explique que le but et le modèle ne se trouvent pas de la même manière chez tous les êtres produits. Pour ce qui est produit à travers le choix, l'art ou la raison, le but de la production est préconçu dans l'esprit de l'agent et est fourni comme le modèle. Il n'en va pas de même pour ce qui est produit par la nature puisqu'elle n'agit ni à travers le choix ni

cine 58 (1984), 315-24 ; id., "Galen's Philosophical Testament : On My Own Opinions", in Jürgen Wiesner (éd.), Aristoteles: Werk und Wirkung (Berlin, 1987), II, 27-51, ici 45-51.

42) Aristote, De la génération des animaux, II, 1, 734 b 10.

43) Sur Simplicius, voir Ilsetraut Hadot, Le problème du néoplatonisme alexandrin: Hiéroclès et Simplicius (Paris, 1978) ; ead. (éd.), Simplicius : sa vie, son cuvre, sa survie (Berlin, 1987). Sur sa réception à la Renaissance, voir Nardi, Saggi sull'aristotelismo, 365-442 ; Charles Lohr, «Renaissance Latin Translations of the Greek Commentaries on Aristotle ", in Jill Kraye et M. W. F. Stone (éds.), Humanism and Early Modern Philosophy (London, 2000), 24-40.

44) Simplicius, Commentaire sur la Physique, II, 3 (Hermann Diels, Simplicii in Aristotelis physicorum libros quatuor priores commentaria (Berlin, 1882), 310-1 = Barrie Fleet, Simplicius: On Aristotle’s Physics 2 (New York, 1997), 67-8). Sur ce fragment, voir Charles Genequand, "Quelques aspects de l'idée de nature d'Aristote à al-Ghazâlî ", Revue de théologie et de philosophie 116 (1984), 105-29, ici 116-7 ; Paolo Accattino, "Alessandro di Afrodisia e la trasmissione della forma nella riproduzione animale ", Atti della Accademia delle scienze di Torino (Classe di scienze morali, storiche e fllologiche) 122 (1988), 79-94, ici 82-4. 
par la raison. C'est là qu'Alexandre qualifie la nature de "puissance irrationnelle", mais conçue comme le principe introduit dans la matière. Ce principe déterminé produit ce dont il est efficient. Le produit de ce principe devient à son tour agent de ce qui suit et ainsi de suite jusqu'à un but déterminé. Alexandre introduit ensuite l'exemple des automates comme le font Aristote et Galien. Nous comprenons donc pourquoi Leoniceno croit que Galien visait Alexandre dans sa critique. Quoi qu'il en soit, Alexandre pense que ce n'est pas par une raison ou un choix, donné aux pièces des automates que leur mouvement est réalisé. Pour lui, il en va de même pour la nature de la semence.

Suite à l'opinion d'Alexandre, Leoniceno donne directement la réponse de Simplicius ${ }^{45}$. Celui-ci se demande, si la génération s'effectue de la manière décrite par Alexandre, comment les choses imparfaites peuvent produire les choses parfaites, par exemple un arbre entier à partir d'une semence. Selon Simplicius, pour qu'il y ait une forme, il faut d'abord une cause générale qui précède les causes particulières. Il ajoute que ce qui est en puissance est amené en acte par autre chose qui est en acte. Comme la cause efficiente immédiate est la nature du père et de la mère chez les animaux, la forme en acte doit préexister chez le père et chez la mère. Simplicius pense donc que la "raison " de l'enfant a préalablement été conçue dans l'homme et, grâce à elle, l'enfant est produit, le père donnant le début du mouvement à travers la semence tout comme les automates mus par une corde. Alors Leoniceno cite la phrase de Simplicius qui met en cause le propos d'Alexandre :

Mais pourquoi appelle-t-il [Aristote selon Alexandre] "puissance irrationnelle » la nature qui agit en vue d'un but et avance en ordre selon les nombres et les mesures préfixées? Est-ce qu'il y a une raison efficiente double dont l'une produit avec la connaissance (c'est elle seule que l'interprète [Alexandre] considère comme la raison) et dont l'autre produit sans connaissance et sans auto-contemplation mais encore avec ordre et détermination en vue d'un but principal ? Et comme ce qui ne connaît pas est appelé "irrationnel " en regard de la raison

45) Simplicius, Commentaire sur la Physique, II, 3 (Diels, 312-4 = Fleet, 69-71), sur Aristote, Physique, II, 3, 194 b 26-9. Cf. Genequand, "Quelques aspects de l'idée de nature ", 118-20. 
connaissante, de même, ce qui produit au hasard et sans ordre est irrationnel s'il est comparé à ce qui agit avec ordre et détermination en vue de quelque chose ${ }^{46}$.

Simplicius semble ainsi distinguer deux sortes de "raisons". La première produit avec connaissance. C'est seulement pour cette espèce qu'Alexandre accepte le titre de "raison ». Elle est, pour ainsi dire, la raison connaissante ou rationnelle. La seconde espèce, en revanche, produit sans connaissance mais avec l'ordre et la détermination. L'agent, qui ne connaît pas ce qu'il produit même s'il le produit avec l'ordre et en vue d'un but, est appelé «irrationnel » par Alexandre. Selon Simplicius, le deuxième mode est celui de la génération animale puisque la nature produit sans choix ce qui lui est similaire par son être tout comme l'anneau produit un sceau. Mais il n'est pas d'accord avec Alexandre pour refuser le titre de « raison " à la nature. C'est pourquoi il insiste sur le fait qu'Aristote appelle une fois la nature « raison » dans le traité De la génération et de la corruption, II, $6^{47}$. Mais comment y a-t-il dans la production un ordre et un but déterminés sans que l'agent ne possède la connaissance? Simplicius répond que les choses naturelles disposent d'une telle constitution qu'elles conservent par leur être l'ordre et la série sans aucune connaissance et qu'elles s'achèvent dans un but déterminé comme les automates. Mais s'il est absurde de dire que les choses naturelles sont produites par hasard, il faut qu'elles s'établissent comme telles par elles-mêmes ou par une autre cause. Ainsi arrive la conclusion de Simplicius que Leoniceno nous délivre :

C'est pourquoi il est raisonnable de dire plutôt que la nature est la "concause " et que les causes prochaines des choses qui naissent et qui se corrompent sont les mouvements des corps célestes suivant lesquels les êtres d'ici-bas s'altèrent tandis que les causes supérieures sont les raisons de ces mouvements, situées dans l'âme [du monde], et les causes encore supérieures à celles-ci sont les formes intellectuelles hors desquelles la lumière formelle est d'abord prélevée pour toutes choses selon la nature et l'aptitude du receveur ${ }^{48}$.

46) Simplicius, Commentaire sur la Physique, II, 3 (Diels, 313 = Fleet, 70-1).

47) Aristote, De la génération et de la corruption, II, 6, 333 b 11.

48) Simplicius, Commentaire sur la Physique, II, 3 (Diels, 314 = Fleet, 71). 
Au lieu d'appeler la nature "raison" quelconque, Simplicius préfere

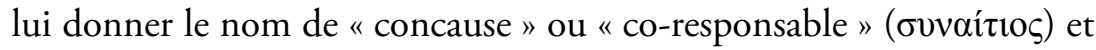
la concevoir comme auxiliaire des causes célestes et intellectuelles. Il faut dire que ce passage lui-même, qui montre une dimension particulièrement néoplatonicienne, est digne d'intérêt. Mais ce qui est plus pertinent pour notre point de vue, c'est que Leoniceno place ce passage au cœur de sa réflexion philosophique sur la vertu formatrice. Il affirme que Simplicius, comme Galien, considère absurde d'attribuer la génération animale à la seule nature qu'Alexandre concevait comme la puissance irrationnelle. Selon lui, Simplicius préfère appeler cette nature "concause » ou " cause instrumentale » tout en admettant l'intervention des causes supérieures et plus puissantes (âme, intellect et formes intellectuelles ou Idées). Pourtant il faut dire que l'intention de Leoniceno est toujours la même. Il s'agit pour lui de réfuter ceux qui croient qu'Aristote identifie la vertu formatrice à l'intellect. En effet, le Stagirite distingue soigneusement dans la Physique entre les choses produites par la nature et celles qui sont produites par l'intellect. Ainsi Leoniceno dit :

Aristote, en traitant de la génération naturelle dans la sphère naturelle, n'en fait aucune mention. Mais plutôt, en voulant ne pas aborder une cause séparée [du corps] dans le deuxième livre De la lecture naturelle, de peur de mélanger la doctrine théologique avec la physique, il a nié que la nature agisse par la volonté et par la raison et a parlé d'elle en tant que puissance irrationnelle. Alexandre a aussi suivi ce sens des mots dans son interprétation. Quant à Simplicius, qui a désiré, autant qu'il a pu, mettre Aristote en accord avec Platon, pour ne pas voir les œuvres de la nature être faites sans aucune connaissance voire par hasard, il leur a donné comme causes non seulement la nature et le ciel mais surtout l'âme et les formes intellectuelles et a ainsi répondu au doute de Galien ${ }^{49}$.

49) De virtute, f. $5 \mathrm{r}=$ ff. $90 \mathrm{r}-90 \mathrm{v}$ : De quo tamen Aristoteles generationem naturalem naturaliter tractans, nullam facit mentionem : quin potius secundo libro De naturali auscultatione nolens causam separatam attingere, ne doctrinam theologicam cum physica confunderet, naturam negavit consilio agere ac ratione, et de ea tamquam de potentia irrationali locutus est. Quem verborum sensum etiam in sua expositione secutus est Alexander. Simplicius vero, qui quantum potuit Platoni Aristotelem studuit facere consentientem, ne naturae opera sine cognitione aliqua et veluti casu facta viderentur, illis non modo naturam ac cœlum, sed praeterea animam et formas intellectuales pro causis arrogavit, atque ita Galeni dubitationi satisfecit. 
Pour Leoniceno, bien qu'elle soit appelée " concause " par Simplicius, la nature reste la cause de la génération animale. Il pense que, par la phrase "Le mouvement de la nature dans le produit même est venu d'une autre nature qui possède une forme en acte ", Aristote parlait de la nature qui réside dans la semence pour la génération animale ${ }^{50}$. Mais il admet que cette phrase laisse encore un doute sur l'identité même de la nature. Pour lui, qu'elle soit l'âme, la vertu psychique, la nature ou la puissance naturelle irrationnelle, celle qui amène une chose moins parfaite à l'état plus parfait doit être la cause de la génération. Alors, en se fondant sur le commentaire de Simplicius sur les Catégories d'Aristote, il essaie de montrer qu'il y a dans la génération une puissance qui réalise cette transition ${ }^{51}$. Selon Leoniceno, Simplicius parle de la puissance naturelle qui est qualifiée de "principe en partie passif et en partie actif " comme espèce secondaire de la qualité. De plus, chez Simplicius, la cause efficiente de la génération des choses naturelles convient à la nature productrice parmi diverses définitions de la nature $^{52}$. Leoniceno précise que la "nature " conçue de telle façon est différente de l'âme. Car l'âme est le principe actif tandis que la nature est le principe passif du mouvement des corps. Il cite ensuite le commentaire sur la Physique, II, 2, où Simplicius explique que, si la nature était le principe actif du mouvement, il n'y aurait pas de différence entre la nature et l'âme. Et il donne la conclusion de Simplicius comme suit :

\begin{abstract}
Mais parce que les corps, largement éloignés de la nature indivisible et incorporelle ainsi que de celle qui établit la vie selon son être, ont été faits comme choses mortes et ne respiraient pas du tout par eux-mêmes étant [trop] refroidis pour toute sorte de vie, ils auront eu en eux une dernière espèce de vie, ce qui est selon la puissance et l'aptitude celle que nous appelons « nature ". Grâce à elle, les choses mortes peuvent aussi être mues et changées, et on dit même qu'elles naissent et agissent en quelque sorte passivement entre elles ${ }^{53}$.
\end{abstract}

50) Aristote, De la génération des animaux, II, 1, 735 a 3-4.

51) Simplicius, Commentaire sur les Catégories, 8 (Karl Kalbfleisch, Simplicii in Aristotelis categorias commentarium (Berlin, 1907), 248 et 249 = Barrie Fleet, Simplicius: On Aristotle's Categories 7-8 (New York, 2002), 108 et 109).

52) Simplicius, Commentaire sur la Physique, II, 2 (Diels, 284 = Fleet, 39). Cf. Hadot, Le problème du néoplatonisme, 177.

53) Simplicius, Commentaire sur la Physique, II, 2 (Diels, 287 = Fleet, 42). Sur la dif- 
Sur la base de ce passage d'une inspiration néoplatonicienne, Leoniceno affirme que la nature, qui est le principe du mouvement dans la semence pour la génération animale, n'est ni l'âme ni la vertu psychique mais la vertu naturelle. C'est là la définition de la vertu formatrice d'Aristote qui émerge de l'interprétation de Simplicius sur la nature productrice. Ainsi, pour Leoniceno, la nature differe de l'âme et est inférieure à l'âme et ce qu'Alexandre appelle "nature " comme premier principe confié à la matière est une puissance qui aide l'introduction de l'âme dans la matière chez les êtres animés.

\section{Averroès et Thémistius : idées, intellect et âme}

Après avoir ainsi éclairci la différence entre les avis d'Alexandre et de Simplicius, Leoniceno critique l'argument d'Averroès exposé dans son Grand commentaire sur la Métaphysique d'Aristote. Il s'agit là d'un texte qui vise à expliquer la célèbre phrase du Stagirite : "C’est un homme qui engendre un homme ${ }^{54}$. Donnons d'abord le propos du passage que cite Leoniceno ${ }^{55}$. Selon Averroès, chez les êtres qui se reproduisent par les semences, celles-ci donnent les formes à ces êtres à travers les formes que les générateurs fournissent aux semences. Chez les êtres qui naissent spontanément sans semence, les corps célestes leurs donnent quelque chose qui joue le rôle des semences et des vertus qui résident dans les semences. Averroès affirme que ces vertus divines engendrent des êtres mutuellement similaires comme les arts produisent leurs produits. C'est pourquoi ces vertus sont comparées à l'intellect puisqu'elles

férence entre la nature et l'âme végétative chez Simplicius, voir Hadot, Le problème du néoplatonisme, 175-8 et 196-8.

54) Aristote, Métaphysique, VII, 9, 1034 b 2. Cf. David M. Balme, «Human is Generated by Human ", in The Human Embryo, 20-31.

55) Averroès, Grand commentaire sur la Métaphysique (désormais indiqué comme GCM), VII, 31 (éd. Giunta, Venise, 1652, VIII, f. 181D-G = Maurice Bouyges, Averroès : Tafsìr mâ ba'd at-tabîat (Beyrouth, 1938-48), 883-4 = Ahmed Elsakhawi, Étude du livre Zây (Dzêta) de la Métaphysique d'Aristote dans sa version arabe et son commentaire par Averroès (Lille, 1994), 116-7). Sur l'impact de cet argument au Moyen Age, voir Charles Touati, "Les problèmes de la génération et le rôle de l'intellect agent ", in Jean Jolivet (éd.), Multiple Averroès (Paris, 1978), 157-64 ; Davidson, Alfarabi, Avicenna, and Averroes, 242-5. 
exercent des actions intellectuelles qui n'ont besoin d'aucun instrument corporel. Ainsi, selon Averroès, ces vertus génératrices que les médecins appellent "formatrices " diffèrent des vertus naturelles du corps animal qui n'agissent qu'à travers des instruments déterminés. En citant la célèbre phrase de Galien : " Je ne sais pas si cette vertu est le Créateur ou non " ${ }^{56}$, Averroès précise que cette vertu, qui agit avec la seule aide de la chaleur séminale, réside dans la semence au titre de forme, non pas comme l'âme dans la chaleur innée des animaux mais comme l'âme dans les corps célestes. C'est pourquoi, conclut-il, Aristote célèbre cette vertu en la rangeant parmi les principes divins.

Leoniceno s'étonne surtout qu'Averroès attribue un tel caractère intellectuel à la vertu formatrice sous l'autorité d'Alexandre qui considérait, en revanche, la nature séminale comme une puissance irrationnelle. Il est donc impossible qu'Alexandre appelle cette vertu " intellect » ou " intellect agent ». D'ailleurs, si Galien compare la vertu formatrice au Créateur, ce n'est pas parce qu'elle agit sans aucun instrument mais parce qu'elle réalise ses œuvres avec une habileté suprême qui semble transcender toutes forces naturelles. Leoniceno reproche donc à Averroès de concilier Galien avec Aristote et Alexandre sur cette base erronée. Pour lui, Galien se positionne loin de ces philosophes qui considèrent la nature comme une puissance irrationnelle. Ainsi, à ses yeux, Averroès comprend mal la position d'Alexandre et allègue à tort sa parole comme si celui-ci partageait le même avis que Galien sur la vertu formatrice.

Leoniceno critique Averroès sur un autre point. Selon lui, Averroès estime faussement que Thémistius est en accord avec Avicenne à propos de la vertu formatrice en s'appuyant sur la célèbre théorie du "donateur des formes" (dator formarum), qui est l'intelligence agente $^{57}$. Pour montrer la faute d'Averroès, il a recours à la paraphrase

56) Cette phrase vient de Galien, De placitis Hippocratis et Platonis, IX, 8 (K, V, $789=$ De Lacy, 597). Cf. Galien, De sententiis, 2 et 11 (Vivian Nutton, Galen : On My Own Opinions (Berlin, 1999), 62 et 90).

57) Averroès, GCM, VII, 31 (Giunta, f. $181 \mathrm{~B}$ = Bouyges, 882-3 = Elsakhawi, 115). Cf. Averroès, GCM, XII, 18 (Giunta, ff. 304 B et $\mathrm{G}$ et $305 \mathrm{~F}=$ Bouyges, 1496, 1498 et 1503 = Aubert Martin, Averroès : Grand commentaire de la Métaphysique d'Aristote, livre lam-lambda (Paris, 1984), 131, 135 et 139). Sur le "donateur des formes" (wâhib al-șwar), voir Amélie-Marie Goichon, La distinction de l'essence et de l'exis- 
de Thémistius sur le De l'âme, I, 3, d'Aristote. Là, Thémistius explique que, si le corps est l'instrument de l'âme, chacune des âmes bâtit un instrument qui lui convient. Elle dessine et prépare d'abord pour elle une maison qui n’a été ni dessinée ni préparée auparavant pour y entrer par la suite ${ }^{58}$. Leoniceno estime que cette théorie de Thémistius ne suppose pas l'existence d'un agent séparé du corps comme le «donateur des formes ». Il dit :

En réalité, Thémistius laisse entendre clairement que ce n'est pas un agent séparé mais l'âme elle-même qui forme pour elle un corps dans lequel elle entre. D'où, il semble avoir estimé que la semence paternelle qui bâtit et fabrique est animée selon Aristote, puisque son âme est celle qui façonne pour elle sa maison à partir de la matière que fournit la femelle. En effet elle n'est ni l'âme du père générateur (autrement elle émigrerait de corps en corps), ni, pour la même raison, l'âme détachée qui doit, selon Thémistius, donner la structure, le trait et l'âme à ces êtres qui naissent de la nature en putréfaction ${ }^{59}$.

Selon Leoniceno, un agent séparé, nécessaire pour la génération spontanée, n'est pas requis chez les êtres vivants qui se reproduisent par la semence puisque l'âme introduite dans la semence par le parent suffit pour former la matière adéquate chez Thémistius. De plus, l'idée que la semence paternelle animée joue le rôle de l'architecte est conforme à l'enseignement d'Aristote. Leoniceno précise toutefois que cette âme réside en puissance dans la semence comme un géomètre endormi ou éveillé, et non comme un géomètre opérationnel ${ }^{60}$. Il conclut donc que Thémistius n'a pas placé la vertu formatrice dans l'âme séparée du

tence d'après Ibn Sînâ (Paris, 1937), 301-3 et 473 ; ead., Lexique de la langue philosophique d'Ibn Sînâ (Paris, 1938), 440-1 ; Davidson, Alfarabi, Avicenna, and Averroes, passim.

58) Thémistius, Paraphrase du De l'âme, I, 3 (Heinze, 23 = Barbaro, f. $74 \mathrm{r}=$ Todd, 40).

59) De virtute, f. $5 \mathrm{v}=$ f. $91 \mathrm{v}$ : Themistius vero non agens separatum, sed animam ipsam formare sibi corpus quod subit, liquido insinuat. Unde videtur sensisse semen maris, quod architectatur et fabricat secundum Aristotelem esse animatum, quando ejus anima est illa quae ex materia, quam fomina praebet, suum sibi facit domicilium. Neque enim est anima patris generantis, alioquin migraret a corpore in corpus, neque ob eandem rationem anima abstracta, quam secundum Themistium oportet his quae ex putrida natura generantur, dare creationem, lineationem, et animam.

60) Cf. Aristote, De la génération des animaux, II, 1, 735 a 10-1. 
corps, comme Averroès le croit à tort, mais dans la semence paternelle qui est animée en puissance. Cela veut dire que, chez Thémistius, la vertu formatrice est identifiée à l'âme dans la semence.

À partir d'ici, Leoniceno exhorte ses lecteurs à rassembler euxmêmes les opinions des anciens commentateurs d'Aristote sur la vertu formatrice, qu'elle soit une puissance naturelle (irrationnelle ou coresponsable) comme chez Alexandre et Simplicius ou l'âme de la semence comme chez Thémistius. Or Averroès lui-même rapporte plusieurs opinions des Arabes dans son commentaire de la Métaphysique, XII, 18. Leoniceno rejette toutefois celle qu'Averroès donne comme sa propre opinion. Car celui-ci la présente comme celle d'Aristote, bien qu'elle soit largement contaminée par la doctrine de Platon. Il dit :

De fait, après qu'il [Averroès] ait écrit beaucoup de choses exprimées, selon lui, dans les livres Des animaux [d'Aristote] sur la chaleur du soleil et des étoiles ainsi que sur les chaleurs qui, dérivées des chaleurs célestes, engendrent les espèces des animaux, il affirme que ces mêmes chaleurs possèdent une propre capacité [causée] par l'art divin intellectuel qui est similaire à la forme unique de l'art unique et principal auquel sont subordonnés plusieurs arts. Il affirme donc que, suivant cet être intelligent, la nature produit parfaitement et régulièrement une chose bien qu'elle ne soit pas consciente, comme si elle était inspirée des vertus actives et plus nobles qui sont appelées " intelligences ». Il ajoute par la suite : "Mais ces proportions et ces vertus qui sont produites dans les éléments par les mouvements du soleil et des autres étoiles sont celles qui sont, dit Platon, les formes [supérieures...] ». C'est ce que dit Averroès qui, étant platonisant comme je l'ai dit, condamne Platon ${ }^{61}$.

${ }^{61)}$ De virtute, f. 6r = ff. 92r-92v: Nam quum multa scripsisset, quae ait esse in libris De animalibus declarata, de calore solis atque stellarum, et caloribus generantibus species animalium ex caloribus cœlestibus derivatis, ait postmodum eosdem calores propriam habere mensuram ab arte divina intellectuali, quae est similis uni formae unius artis principalis sub qua sunt artes plures. Secundum hoc igitur ait esse intelligentum, quod natura facit aliquid perfecte et ordinate, quamvis non intelligat, quasi esset rememorata ex virtutibus agentibus nobilioribus, quae dicuntur intelligentiae. Postmodum subjungit : Istae autem proportiones et virtutes quae fiunt in elementis a motibus solis, et aliarum stellarum sunt hae, quas reputat Plato esse formas [...]. Haec quidem Averrois, qui platonizans, ut dixi, Platonem damnat. Cf. Averroès, GCM, XII, 18 (Giunta, f. 305 D-E = Bouyges, 1502-3 = Martin, 138-9). 
Leoniceno souligne clairement la contradiction d'Averroès qui, à la fois, s'inspire du platonisme et le condamne. Mais refuse-t-il le recours même au platonisme ? Leoniceno dit que, si la nature est la nature universelle de toutes choses naturelles, elle doit d'abord recevoir les " raisons » de tous les êtres à engendrer et puis rendre ces êtres similaires aux raisons contenues en elle. Ces raisons sont bel et bien les Idées de Platon bien qu'Averroès prétende préférer l'avis d'Aristote. Pour Leoniceno, la différence entre deux opinions est pourtant minime. Car le meilleur modèle est la solution de Simplicius. Il dit :

Ces deux opinions ne sembleront pourtant pas très différentes, si ces causes supérieures au-delà du ciel et de la nature, c'est-à-dire l'intellect excellent et les formes intellectuelles, sont aussi comptées, tout comme lorsque Simplicius résout, à la différence d'Alexandre, d'une manière aussi platonicienne qu'aristotélicienne le doute concernant la génération naturelle et la nature en tant que puissance irrationnelle $e^{62}$.

Leoniceno ajoute que la génération spontanée se réalise effectivement chez Thémistius grâce à l'âme du monde véhiculée par la nature entière et qu'Averroès accepte également cette idée qui n'est pas discordante avec la théorie d'Aristote exposée dans le GA, III, $11^{63}$. Nous remarquons donc que Leoniceno ne condamne pas le recours même au

62) De virtute, f. $6 \mathrm{r}=$ f. $92 \mathrm{v}$ : Quae tamen duae opiniones non multum videbuntur discrepare, si praeter coelum atque naturam causae etiam illae superiores, mens scilicet eximia et formae intellectuales, adhibeantur : Quando et Simplicius tactam contra Alexandrum de generatione naturali, et natura, potentia irrationali dubitationem, non magis Platonice, quam etiam Aristotelice solvit.

63) Aristote, De la génération des animaux, III, 11, 762 a 18-21. Sur la génération spontanée, voir Hiro Hirai, "Earth's Soul and Spontaneous Generation : Fortunio Liceti's Criticism against Ficino's Ideas on the Origin of Life ", in Stephen Clucas et al. (eds.), Laus Platonici Philosophi : Marsilio Ficino and His Influence (Leyde : Brill, à paraître). Sur l'âme du monde chez Thémistius, voir ses Paraphrase du De l'âme, I, 3 et 4 (Heinze, 20 et $26=$ Barbaro, ff. $73 \mathrm{r}$ et $74 \mathrm{v}=$ Todd, 36 et 42-3); Paraphrase de la Métaphysique, XII, 3 (Samuel Landauer, Themistii in Aristotelis metaphysicorum librum XII paraphrasis (Berlin, 1903), 9 = Rémi Brague, Thémistius: Paraphrase de la Métaphysique d'Aristote (Livre Lambda) (Paris, 1999), 64). Sur ce dernier traité, voir Shlomo Pinès, "Some Distinctive Metaphysical Conceptions in Themistius' Commentary on Book Lambda and their Place in the History of Philosophy ", in Aristoteles : Werk und Wirkung, II, 177-204 ; Brague, Thémistius. 
platonisme. Ce qu'il n'accepte pas, c'est l'identification de la vertu formatrice avec l'intellect.

Enfin Leoniceno trouve une répercussion importante de l'interprétation averroïste chez Gentile da Foligno (?-1348), commentateur emblématique du Canon d'Avicenne ${ }^{64}$. En effet, Gentile, qui suit fidèlement Averroès, considère qu'il faut supposer dans les êtres naturels quelque chose qui agit à travers l'intellect pour introduire la forme dans la matière. Leoniceno cite sa phrase comme suit :

[...] mais nous voyons dans l'art qu'il y a là quelque chose qui agit à travers l'intellect en limitant, traitant et préparant la matière, comme il est évident, par exemple, dans l'art artisanal. De fait, il existe chez l'ouvrier un certain art cognitif qui limite, mesure, traite et prépare la matière [...]. Il y aura ainsi de même dans la nature, évidemment, quelque chose qui agit à travers l'intellect en limitant, traitant et préparant la matière. Et le corps céleste tout entier, composé de tout, produit ceci en tant qu'instrument par son mouvement et par sa lumière principalement à travers les [causes] intellectuelles qui sont en lui et dans lesquelles cet art a été reçu à l'intérieur. Cet art productif est [celui] de toute forme inférieure ou de ce qui existe ici-bas. D'où, Averroès disait dans le douzième livre de la Métaphysique que la nature n'agit pas si elle n'est pas inspirée des causes supérieures et plus divines ${ }^{65}$.

Leoniceno critique Gentile surtout parce que celui-ci affirme, suite à cet argument, que tous les disciples d'Aristote sont en accord. Mais, comme nous l'avons vu, Alexandre n'est pas du tout du même avis.

64) Sur Gentile, voir Roger French, "Gentile da Foligno and the Via medicorum », in John D. North et John J. Roche (éds.), The Light of Nature: Essays in the History and Philosophy of Science Presented to A.C. Crombie (Dordrecht, 1985), 21-34 ; id., Canonical Medicine: Gentile da Foligno and Scholasticism (Leyde, 2001) ; Joël Chandelier, "Gentile da Foligno et le mouvement du cour ", Micrologus 11 (2003), 97-122.

65) De virtute, $\mathrm{f} .6 \mathrm{r}=\mathrm{ff} .92 \mathrm{v}-93 \mathrm{r}:[\ldots]$ sed in arte videmus, quod est ibi aliquod agens per intellectum limitans, agens, praeparans materiam: sicuti verbi gratia in arte fabrili apparet: Nam in fabro est ars quaedam cognoscitiva limitans, et mensurans, agens, praeparans materiam [...]. Sic igitur etiam erit in natura, scilicet agens aliquod per intellectum limitans, agens, paraeparans materiam. Et totum corpus cceleste compositum ex omnibus, quod quidem hoc efficit instrumentaliter per motum et lumen principaliter per intellectivas, quae in eo sunt in quibus recepta est ars quae intro. Quae quidem ars factiva est omnis formae inferioris vel inferius existentis. Unde dicebat Averrois duodecimo Metaphysices, quod natura non operatur nisi reminiscens ex superioribus causis divinioribus. Cf. Averroès, GCM, XII, 18 (Giunta, f. 305 D-E = Bouyges, 1502-3 = Martin, 139). 
Alors il conclut que, au lieu d'attribuer à Aristote de telles idées qui sont plutôt de Platon, Simplicius a beaucoup plus correctement montré ce que le Stagirite aurait dû dire si l'on voulait parler des choses conformes à ses enseignements.

\section{Conclusions}

Nous avons tracé jusqu'ici les grandes lignes de la discussion de Leoniceno et analysé les sources et les thèmes pertinents qu'il développe autour de la notion de vertu formatrice. Il critique les Médiévaux, notamment Averroès et Pietro d'Abano, en s'appuyant largement sur les nouvelles traductions humanistes (y compris les siennes) d'Aristote et des commentateurs grecs comme Alexandre, Thémistius et Simplicius ou sur les textes jusqu'alors méconnus et non traduits en latin comme ceux de Galien et de Michel d'Éphèse. Il faut surtout souligner son recours aux anciens commentateurs grecs puisque Leoniceno est l'un des premiers auteurs à les avoir impliqués dans les discussions de la philosophie naturelle et médicale de cette époque. De cette manière, il fournit pour la première fois aux lecteurs occidentaux les opinions étrangères à la tradition arabo-latine sur la nature de la vertu formatrice. Nous pouvons donc dire que son traité De virtute formativa se trouve au «carrefour » de la tradition médiévale et de la nouvelle tendance humaniste propre à la Renaissance. Il ouvre ainsi une nouvelle voie dans les spéculations embryologiques du seizième siècle. Ce qui anime l'esprit de Leoniceno, c'est un anti-arabisme et un amour pour les sources grecques, amour qui se traduit à travers sa méticulosité philologique ${ }^{66}$.

Quant à la véritable identité de la vertu formatrice, Leoniceno se fait simplement l'interprète de chaque auteur. Plutôt que d'établir sa propre synthèse, il préfere souligner en philologue le véritable sens des termes et des phrases et les contradictions et les cohérences propres à l'argumentation de chaque auteur ou de son interprète. Notre analyse

66) Sur l'anti-arabisme, voir Gerhard Baader, "Medizinisches Reformdenken und Arabismus im Deutschland des 16. Jahrhunderts ", Sudhoffs Archiv 63 (1979), 26196, ici 270-3 ; Nancy G. Siraisi, Avicenna in Renaissance Italy: The Canon and Medical Teaching in Italian Universities after 1500 (Princeton, 1987), 66-77. 
a toutefois montré qu'il préfere l'interprétation néoplatonicienne de Simplicius. Ce que Leoniceno refuse pertinemment, c'est l'identification de la vertu formatrice avec l'intellect. Cette identification, qu'il estime fausse, est souvent introduite quand la production artificielle et la génération naturelle sont confondues par analogie comme chez Averroès, Pietro et Gentile.

Nous avons aussi observé combien Leoniceno était sensible aux textes qui montrent des éléments néoplatoniciens, non seulement chez Simplicius mais aussi chez Thémistius. À ce propos, sa connaissance des idées platoniciennes du cardinal Bessarion n'est sans doute pas le fruit de hasard. Son développement sur le véhicule de l'âme, thème vénéré des Néoplatoniciens florentins tels que Marsile Ficin (14331499), nous montre également sa familiarité avec les débats contemporains des humanistes.

Enfin la discussion principalement embryologique de Leoniceno, qui aborde aussi les problèmes philosophiques importants de l'âme et de l'intellect, coïncide avec le développement du nouvel aristotélisme de ses contemporains padouans comme Agostino Nifo (1473-ca. 1538) ou Pietro Pomponazzi (1462-1525) qui utilisaient amplement les écrits des anciens commentateurs grecs ${ }^{67}$. Il serait légitime de se demander s'il n'y avait pas d'éventuels échanges d'idées entre eux et Leoniceno. Cette remarque n'est peut-être pas inutile pour mieux comprendre les aspects de l'aristotélisme renaissant, qui ont été étudiés jusqu'à présent presque exclusivement dans les domaines de la philosophie. Mais ce serait sans doute le sujet à approfondir dans une autre étude.

67) Cf. Nardi, Saggi sull'aristotelismo, passim ; id., Studi su Pietro Pomponazzi (Florence, 1965) ; Poppi, Causalità e infinità, 222-36 ; Martin L. Pine, Pietro Pomponazzi : Radical Philosopher of the Renaissance (Padoue, 1986) ; Edward P. Mahoney, Two Aristotelians of the Italian Renaissance : Nicoletto Vernia and Agostino Nifo (Aldershot, 2000). À Ferrare, Leoniceno était proche du cercle intellectuel d'Alberto Pio de Carpi (1475-1531), cercle auquel se fréquentait Pomponazzi dans les années 1496-9. Voir Cesare Vasoli, La cultura delle corti (Bologne, 1980), 94, 98 et 100-1 ; Charles B. Schmitt, "Alberto Pio and the Aristotelian Studies of his Time", Medioevo et Umanesimo 46 (1981), 43-64, repris dans The Aristotelian Tradition and Renaissance Universities (London, 1984); Stefano Perfetti, Aristotle's Zoology and Its Renaissance Commentators (1521-1601) (Louvain, 2000), 35 et 49-50. 